ARTICLE

Received 16 Feb 2015 | Accepted 18 May 2015 | Published 26 Jun $2015 \quad$ DOl: 10.1038/ncomms8533 OPEN

\title{
Kinetics and fracture resistance of lithiated silicon nanostructure pairs controlled by their mechanical interaction
}

Seok Woo Lee ${ }^{1, \star}$, Hyun-Wook Lee ${ }^{2, \star}$, III Ryu ${ }^{3}$, William D. Nix ${ }^{2}$, Huajian Gao ${ }^{3}$ \& Yi Cui ${ }^{2,4}$

Following an explosion of studies of silicon as a negative electrode for Li-ion batteries, the anomalous volumetric changes and fracture of lithiated single $\mathrm{Si}$ particles have attracted significant attention in various fields, including mechanics. However, in real batteries, lithiation occurs simultaneously in clusters of $\mathrm{Si}$ in a confined medium. Hence, understanding how the individual Si structures interact during lithiation in a closed space is necessary. Here, we demonstrate physical and mechanical interactions of swelling Si structures during lithiation using well-defined Si nanopillar pairs. Ex situ SEM and in situ TEM studies reveal that compressive stresses change the reaction kinetics so that preferential lithiation occurs at free surfaces when the pillars are mechanically clamped. Such mechanical interactions enhance the fracture resistance of lithiated Si by lessening the tensile stress concentrations in Si structures. This study will contribute to improved design of Si structures at the electrode level for high-performance Li-ion batteries.

\footnotetext{
${ }^{1}$ Geballe Laboratory for Advanced Materials, Stanford University, Stanford, California 94305, USA. ${ }^{2}$ Department of Materials Science and Engineering, Stanford University, Stanford, California 94305, USA. ${ }^{3}$ School of Engineering, Brown University, Providence, Rhode Island 02912, USA. ${ }^{4}$ Stanford Institute for Materials and Energy Sciences, SLAC National Accelerator Laboratory, 2575 Sand Hill Road, Menlo Park, California 94025, USA. * These authors contributed equally to this work. Correspondence and requests for materials should be addressed to Y.C. (email: yicui@stanford.edu).
} 
S ilicon ( $\mathrm{Si}$ ) has attracted great attention as a promising negative electrode material for Li-ion batteries due to its exceptional theoretical specific capacity of $3,578 \mathrm{mAh}^{-1}$ for the $\mathrm{Li}_{15} \mathrm{Si}_{4}$ phase at room temperature ${ }^{1-5}$. Despite these preeminent theoretical properties, conventional $\mathrm{Si}$ anodes face significant challenges due to the large volume changes that accompany lithiation. These effects have limited the choice of Si as a commercial negative electrode because they can lead to the loss of electrical contact between active materials by mechanical fracture, accumulation of solid-electrolyte interphase layers, and rapid capacity fading during electrochemical cycling ${ }^{6-9}$. Recently, nanotechnology has achieved a breakthrough to overcome the aforementioned challenges of $\mathrm{Si}$ as a negative electrode for Li-ion batteries ${ }^{1,2}$. Various Si nanomaterials and engineered Si nanostructures such as nanowires/particles, hollow spheres and porous nanostructures have demonstrated stable cycling and resistance to fracture in spite of the large volume change of $\mathrm{Si}^{1,10-12}$. Engineered nanostructures, wherein the surface of the $\mathrm{Si}$ structure does not strain and where a gap for volume expansion of lithiated $\mathrm{Si}$ is provided, lead to a stable solid-electrolyte interphase layer formation on the surface of the electrode material and enhanced Coulombic efficiency and markedly improved cycle life ${ }^{7,8}$.

Accompanying the search for high-performance $\mathrm{Si}$ anodes, fundamental studies have provided a better idea of how $\mathrm{Si}$ lithiates, swells and fractures, leading to a basis for the rational design of $\mathrm{Si}$ structures ${ }^{4}$. Especially, the extreme volumetric and structural changes of lithiated Si have attracted much attention in mechanics because of the large stress evolution and corresponding mechanical fracture. The marked change of mechanical properties by lithiation has been documented by simulations and experiments ${ }^{13-18}$. Analytical and numerical analyses, including both elasticity and plasticity, have suggested both diffusion-induced stress models and pressurized hollow

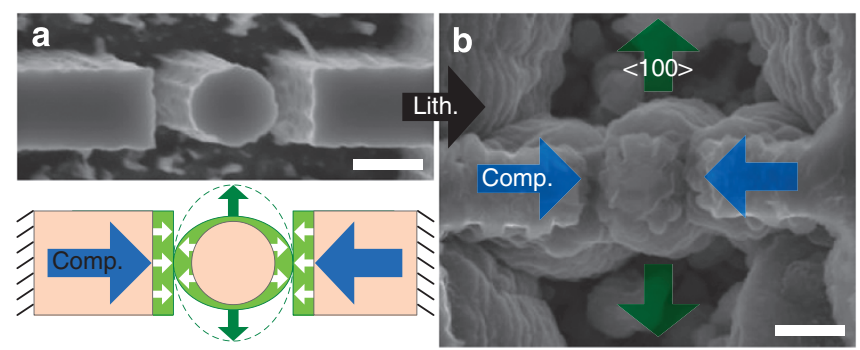

C

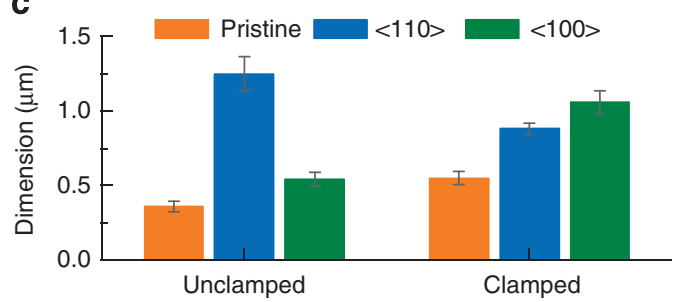

Figure 1 | SEM study of the lithiation of a clamped $<\mathbf{1 1 0}>$ Si nanopillar. $(\mathbf{a}, \mathbf{b})$ SEM images of $<110>$ Si nanopillar positioned between adjacent rigid walls before (a) and after (b) lithiation. The electrochemical lithiation of a single pillar was suppressed by compressive stresses between the two rigid walls, which were supposed to be preferably grown to $<110\rangle$ direction as displayed in a schematic diagram (a). (c) Column chart of dimension change of $<110>$ Si nanopillar along $<110>$ (blue) and $<100>$ (green) direction after lithiation when the pillar is unclamped 30 and clamped. Single $<110>$ Si nanopillar standing alone has preferential lithiation along $<110>$ directions of Si but the clamped Si nanopillar shows further expansion along $<100>$ direction. structure models of lithiation/delithiation of $\mathrm{Si}$ as a part of an effort to explain how the expansion causes stress evolution and mechanical fracture ${ }^{19-24}$. These models are based on experimental observations such as volumetric changes, mechanical fracture and structural changes ${ }^{25-28}$. Recently, top-down fabrication of Si nanostructures allowed the systematic study of the effects of crystal orientation, dimensions and morphology that revealed preferential lithiation along $\langle 110\rangle$ directions of crystalline $\mathrm{Si}$, a size dependence of the fracture resistance, and the robustness of amorphous $\mathrm{Si}^{6,29-33}$. In situ transmission electron microscopy (TEM) has provided time-series crystallographic and chemical information as well as information about the morphology of lithiated $\mathrm{Si}^{34}$. The observed dynamic behaviour of $\mathrm{Si}$ nanostructures provided information about the kinetics of lithiation controlled by mechanical stresses and the orientation of the reaction interface of crystalline $\mathrm{Si}$ as well as the aforementioned anisotropic expansion and fracture behaviour $^{35-39}$.

However, in a real battery system, Si structures form as clusters at the electrode level and the lithiation of the individual structures occurs simultaneously in a confined medium. Then, swelling Si structures in fixed volume mechanically interact with each other and the reaction kinetics and fracture behaviour become more complicated than that observed for single-particle systems. Therefore, understanding how the individual $\mathrm{Si}$ structures mechanically interact during lithiation is necessary for the rational design of Si electrodes. Here we show how mechanical interactions of neighbouring crystalline Si structures affect their reaction kinetics and fracture resistance during electrochemical lithiation, using ex situ scanning electron microscopy (SEM) and in situ TEM of Si nanopillar pairs.

\section{Results}

Lithiation of mechanically clamped Si pillar. To mimic the cluster of crystalline Si particles in the confined volume in the negative electrode of a Li-ion battery, Si nanopillars with adjacent rigid walls were fabricated by e-beam lithography and dry etching of $\langle 110\rangle$ single crystalline $\mathrm{Si}$ wafer (see Methods and Supplementary Fig. 1a-c). We used $<110\rangle$ Si pillars so that lateral volume expansion would occur preferentially along two opposite $\langle 110\rangle$ directions on lithiation. To simulate mechanical clamping of Si structure in closed-packed media, e-beam lithography defined the various diameters of the pillars and the location of rigid walls for two different geometries so that rigid walls block both sides of $<110>$ direction of the pillar. For the ex situ SEM study, the fabricated silicon nanopillar and wall array on a piece of wafer was lithiated by sweeping voltage down to $10 \mathrm{mV}$ versus $\mathrm{Li} / \mathrm{Li}^{+}$and held for more than $10 \mathrm{~h}$ in a half cell with Li foil (see Methods and Supplementary Fig. 1d-i). For the in situ TEM study, the pillars were placed at the edge $<110>$ direction of the piece of $\langle 110\rangle$ wafer and mounted on the TEM holder with a proper tilting so that the pillar can be observed under e-beam without shading (see Supplementary Fig. 2). After building the solid cell configuration with a $\mathrm{Li} / \mathrm{Li}_{2} \mathrm{O}$ counter electrode, the pillar is lithiated by applying d.c. bias during the TEM observation.

To simulate the mechanical clamping of Si structures in closedpacked media, a pillar was prepared between two rigid walls blocking both $<110>$ directions on lithiation as shown in Fig. 1 . A $<110>$ Si pillar $550 \mathrm{~nm}$ in diameter standing between two rigid walls with $320 \mathrm{~nm}$ gaps was fabricated for the SEM study (Fig. 1a). Since the crystal orientation is identical to the first case, the pillar and the walls expand laterally along $<110>$ directions and fill the gap between them on lithiation. After the contact, the lithiation along the $\langle 110\rangle$ direction cannot proceed due to the 
build-up of compressive stresses and the pillar lithiates along a second favoured direction, $\langle 100\rangle$ as shown in Fig. 1b. The walls also expand along the $<100\rangle$ direction after contact with the pillar. Figure 1c compares dimension changes of the unclamped and clamped $\langle 110\rangle$ pillars. The unclamped pillar with a diameter of $0.36 \mu \mathrm{m}$ expands to 1.25 and $0.54 \mu \mathrm{m}$ along $<110\rangle$ and $\langle 100\rangle$ directions, respectively, on lithiation, as found in our previous study ${ }^{30}$. The unclamped pillar clearly shows anisotropic expansion behaviour where the $<110\rangle$ direction exhibits a faster reaction than the $\langle 100\rangle$ direction. In contrast, the clamped pillar with a diameter of $0.55 \mu \mathrm{m}$ expands to 0.88 and $1.06 \mu \mathrm{m}$ along the $<110\rangle$ and $<100\rangle$ directions, respectively, on lithiation. Ideally, the swelling pillar and wall would come into contact in the middle of the gap and the width of the pillar along the $\langle 110\rangle$ direction would then be $0.87 \mu \mathrm{m}$ ( = original diameter $+2 \times$ gap $/ 2$ ), which is indeed very close to the measured width. Therefore, it is clear that the lithiation along the $\langle 110\rangle$ direction is stopped at the point of contact and the subsequent lithiation continues along the $<100>$ direction.

In situ TEM observation of the $<110>$ Si pillar near the wall provides a better picture of the dynamic lithiation behaviour of the crystalline $\mathrm{Si}$ core and the corresponding mechanical interaction. The electron beam penetrates through the $<100>$ direction of the $\langle 110>$ Si nanopillar standing by the rigid wall, so a lateral $\langle 110\rangle$ expansion of the nanopillar can be monitored during the lithiation process (Fig. 2a,b). The reaction stoppage of the pillar after the contact is clearly shown in the in situ TEM study. For the mechanical clamping, three $<110>$ Si nanopillars with the same diameters of $550 \mathrm{~nm}$ and rigid walls on either side of the pillars were fabricated as shown in Fig. 2b. A single pillar clamped by two rigid walls also exhibits termination of the expansion as shown in ex situ SEM, but overlapping of structures hindered precise measurement (see Supplementary Movie 1). The time series of TEM images of the lithiating pillars clamped by the walls are shown in Fig. 2c-e (see also Supplementary Movies 2 and 3). At the beginning of the lithiation, the pillars start to expand as a normal $\langle 110\rangle$ single pillar does in spite of inconsistent expansion due to irregular contact with $\mathrm{Li}$ metal (Fig. $2 \mathrm{c}, \mathrm{d}$ ). After the contact, a $\mathrm{Li}_{x} \mathrm{Si}$ shell fills the empty space and the crystalline $\mathrm{Si}$ core stops shrinking due to the termination of the lithiation (Fig. 2e). The plot of the diameters of the $\mathrm{Li}_{x} \mathrm{Si}$ outer shell and the crystalline $\mathrm{Si}$ core as a function of time clearly shows that the expansion of the shell and shrinkage of the core are slowing down on the contact and halted at about $90 \mathrm{~s}$ (Fig. 2f). Since then, the diameter of the remaining crystalline core is maintained for over $400 \mathrm{~s}$ and the lithiation cannot proceed further along $\langle 110\rangle$ direction against the neighbouring pillars due to the mechanical clamping. In contrast, unclamped pillar exhibits the completed lithiation and the mechanical fracture without the termination of the lithiation (see Supplementary Movies 4 and 5).

Analytical model. To explain how mechanical clamping stops the lithiation at the contact, an analytical model is developed by considering mechanical stress evolution on the clamping and change of driving force of the reaction. The driving force of the lithiation is defined as:

$$
\Delta G=\Delta G_{r}^{\mathrm{Li}_{x} \mathrm{Si}}-e \Phi+\Delta G_{\sigma},
$$

where $\Delta G$ is the change of Gibbs free energy, $\Delta G_{\mathrm{r}}^{\mathrm{Li}} \mathrm{Si}$ is the change of free energy of lithiation without applied voltage or mechanical stress, $\Phi$ is the applied voltage to the electrochemical cell, and $\Delta G_{\sigma}$ is the change of free energy due to mechanical stress ${ }^{21}$. $\Delta G_{\sigma}$ expresses the relationship between mechanical stress at the a
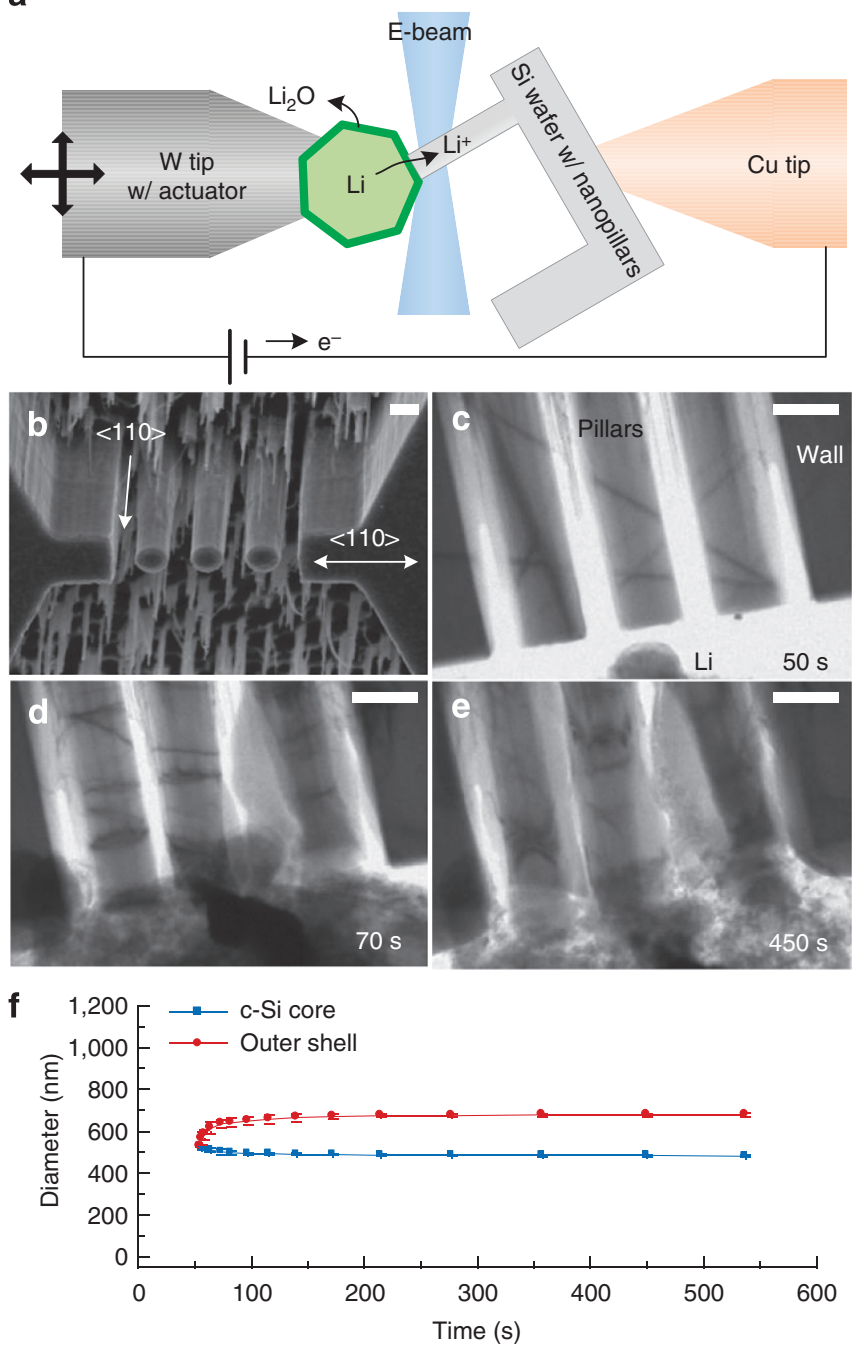

Figure 2 | In situ TEM study of the lithiation of a clamped $<110>$ Si nanopillar. (a) A schematic image of the electrochemical cell configuration for in situ TEM observation. E-beam penetrates through $<100>$ direction of Si nanopillar to observe a lateral $<110>$ expansion during lithiation. (b) SEM image of pristine three pillars with adjacent rigid walls on both sides for in situ TEM observation. (c-e) Time series of TEM images of the pillars during lithiation. All scale bars in SEM and TEM images are $500 \mathrm{~nm}$. (f) The diameters of crystalline $\mathrm{Si}$ core and lithiated outer $\mathrm{Li}_{x} \mathrm{Si}$ for the time line in the middle of lithiation. The lithiation cannot proceed further along $<110>$ direction against the neighboring pillars due to the mechanical clamping.

atomically sharp interface of crystalline $\mathrm{Si}$ and swelling $\mathrm{Li}_{x} \mathrm{Si}$ alloy and the change of the driving force of the reaction ${ }^{28}$. Considering the consumption of one $\mathrm{Li}$ atom to form $1 / x$ units of $\mathrm{Li}_{x} \mathrm{Si}, \Delta G_{\sigma}$ is computed as ${ }^{21,35}$ :

$$
\Delta G_{\sigma}=\frac{1}{x}\left(\sigma_{\mathrm{m}}^{\mathrm{Si}} \Omega^{\mathrm{Si}}-\sigma_{\mathrm{m}}^{\mathrm{Li}_{x} \mathrm{Si}} \Omega^{\mathrm{Li}_{x} \mathrm{Si}}\right),
$$

where $\sigma_{\mathrm{m}}^{\mathrm{Si}}$ and $\sigma_{\mathrm{m}}^{\mathrm{Li}_{x} \mathrm{Si}}$ are the mean stresses in the crystalline $\mathrm{Si}$ and in the $\mathrm{Li}_{x} \mathrm{Si}$ at the interface, respectively, and $\Omega^{\mathrm{Si}}$ and $\Omega^{\mathrm{Li} \mathrm{S}_{x} \mathrm{Si}}$ are the volumes per $\mathrm{Si}$ atom and unit of $\mathrm{Li}_{x} \mathrm{Si}$, respectively. Since a negative $\Delta G$ drives lithiation, compressive hydrostatic stress in the crystalline $\mathrm{Si}$ or tensile hydrostatic stress in the $\mathrm{Li}_{x} \mathrm{Si}$ enhances lithiation process. The model for the estimation of stress on lithiation includes consideration of both the 'Before contact' and 'After contact' of neighbouring Si structures. Figure 3a shows a schematic view of the model of 'Before contact'. A square 
a

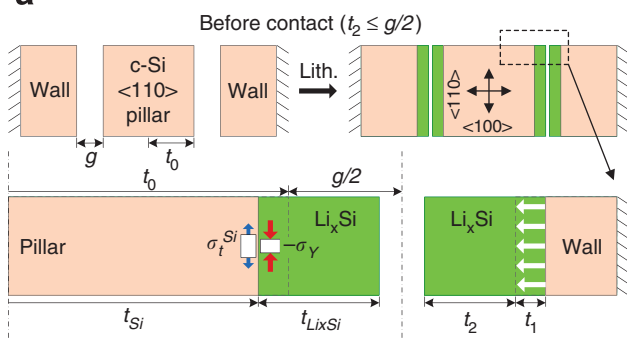

b

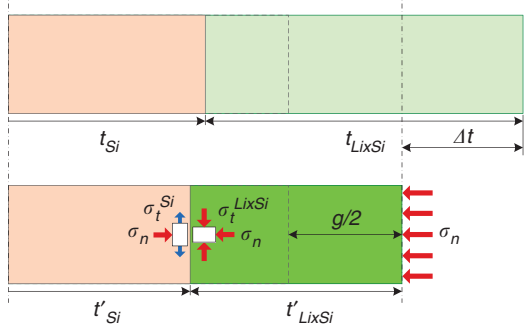

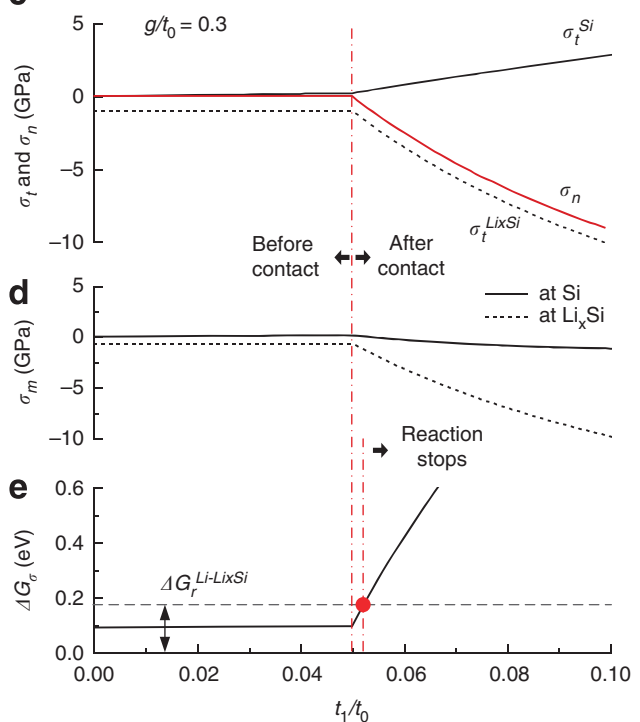

Figure 3 | Analytical model of the clamped Si pillar to predict the change of the driving force of the reaction. (a) A schematic view of $<110>$ crystalline Si with wall fixed at the end. The scheme represents morphological expansion and induced stresses during lithiation of $<110>$ pillars and walls before the physical contact ('Before contact', $t_{2}<g / 2$ ). (b) A schematic view of the one side of Si pillar contacted with the wall physically ('After contact', $t_{2} \geq g / 2$ ). The displacement of lithiated $\mathrm{Si}$ is confined as a half of the gap $(\mathrm{g} / 2)$. (c) Normal $\left(\sigma_{\mathrm{n}}\right)$ and tangential $\left(\sigma_{\mathrm{t}}\right)$ stress at the interfaces in the crystalline $\mathrm{Si}$ and $\mathrm{Li}_{x} \mathrm{Si}$ for the depth of lithiation $\left(t_{\sqrt{ }} t_{0}\right)$ when $g / t_{0}$ is 0.3 . (d) Mean stress $\left(\sigma_{\mathrm{m}}\right)$ at the interfaces in the crystalline $\mathrm{Si}$ (solid) and $\mathrm{Li} \mathrm{i}_{x} \mathrm{Si}$ (dotted) for the depth of lithiation $\left(t_{\sqrt{ }} t_{0}\right)$ when $g / t_{0}$ is 0.3 . (e) Corresponding change of free energy due to mechanical stress $\left(\Delta G_{\sigma}\right)$ for the depth of lithiation $\left(t_{\sqrt{ }} t_{0}\right)$ when $g / t_{0}$ is 0.3 . Black dash line represents free energy of Li deposition versus free energy of lithiation of $\mathrm{Si}\left(\Delta G_{r}^{\mathrm{Li}-\mathrm{Li}_{\times} S \mathrm{i}}\right)$. Red vertical lines indicate the contact and reaction stoppage on lithiation of $\mathrm{Si}$, respectively.

$<110>$ Si pillar of $2 t_{0}$ width is located between two fixed rigidwall structures aligned along the lateral $\langle 110\rangle$ direction with a gap of $g$. Assuming dominant expansion and propagation of flat $\{110\}$ interface along the $\langle 110\rangle$ direction as shown in the previous studies ${ }^{31}$, after lithiation the thicknesses of crystalline $\mathrm{Si}$ core and the $\mathrm{Li}_{x} \mathrm{Si}$ layer may be called $t_{\mathrm{Si}}$ and $t_{\mathrm{Li}_{x} \mathrm{Si}}$, respectively. Also $t_{1}\left(=t_{\mathrm{Li}_{\mathrm{x}} \mathrm{Si}} / 4\right)$ is the thickness of the consumed crystalline $\mathrm{Si}$ and $t_{2}$ is the displacement of each surface towards each other. Before the structures contact each other, $\mathrm{Li}_{x} \mathrm{Si}$ in the gap is free to expand laterally along the $\langle 110\rangle$ direction, which is normal to the interface, so the normal stress $\left(\sigma_{\mathrm{n}}\right)$ is zero. The tangential biaxial stress at the interface in $\mathrm{Li}_{x} \mathrm{Si}\left(\sigma_{t}^{\mathrm{Li}_{x} \mathrm{Si}}\right)$ is equal to the compressive yield strength $\left(-\sigma_{Y}\right)$ assuming plastic deformation in the lithiated $\mathrm{Si}^{21}$. Before the contact, mechanical equilibrium requires the tangential biaxial stress at the interface in crystalline $\mathrm{Si}\left(\sigma_{t}^{\mathrm{Si}}\right)$ to be related to $\sigma_{t}^{\mathrm{Li}_{x} \mathrm{Si}}$ and the ratio of the thickness of $\mathrm{Li}_{x} \mathrm{Si}$ $\left(t_{\mathrm{Li}_{x} \mathrm{Si}}\right)$ to the half thickness of crystalline $\mathrm{Si}\left(t_{\mathrm{Si}}\right)$, as follows:

$$
\sigma_{t}^{\mathrm{Si}}=\frac{t_{\mathrm{Li}_{x} \mathrm{Si}}}{t_{S i}} \sigma_{\mathrm{Y}}=\frac{4 t_{1}}{t_{0}-t_{1}} \sigma_{\mathrm{Y}}=\frac{4 t_{1} / t_{0}}{1-t_{1} / t_{0}} \sigma_{\mathrm{Y}}
$$

Then, the mean stresses at the interfaces in the crystalline Si core and in the $\mathrm{Li}_{x} \mathrm{Si}$ layer are expressed as:

Before contact:

$$
\sigma_{\mathrm{m}}^{\mathrm{Si}}=\frac{\sigma_{\mathrm{t}_{1}}^{\mathrm{Si}}+\sigma_{\mathrm{t}_{2}}^{\mathrm{Si}}}{3}=\frac{2 \sigma_{\mathrm{t}}^{\mathrm{Si}}}{3}, \quad \sigma_{\mathrm{m}}^{\mathrm{Li}_{x} \mathrm{Si}}=\frac{\sigma_{\mathrm{t}_{1}}^{\mathrm{Li}_{\mathrm{x}} \mathrm{Si}}+\sigma_{\mathrm{t}_{2}}^{\mathrm{Li}_{x} \mathrm{Si}}}{3}=-\frac{2 \sigma_{\mathrm{Y}}}{3}
$$

where $\sigma_{t_{1}}^{\mathrm{Si}}=\sigma_{t_{2}}^{\mathrm{Si}}=\sigma_{t}^{\mathrm{Si}}$ and $\sigma_{t_{1}}^{\mathrm{Li}_{x} \mathrm{Si}}=\sigma_{t_{2}}^{\mathrm{Li}_{x} \mathrm{Si}}=\sigma_{t}^{\mathrm{Li}_{x} \mathrm{Si}}=-\sigma_{\mathrm{Y}}$. After contact, the displacement of each of the two surfaces is limited to half of the initial gap, $g / 2$ and a normal stress at the interface, $\sigma_{\mathrm{n}}$ develops on the $\{110\}$ interface in crystalline $\mathrm{Si}$ and on the $\mathrm{Li}_{x} \mathrm{Si}$ layer (Fig. 3b). Since the deformation is fully constrained by the contact and the interfacial compatibility, additional plastic deformation is no longer possible and additional lithiationinduced strain must be accommodated by the elastic deformation. In this case, the stress state in the $\mathrm{Si}$ core and $\mathrm{Li}_{x} \mathrm{Si}$ layer can be computed by the superposition of the normal stress. The tangential stress at the interface in $\mathrm{Li}_{x} \mathrm{Si}$ is then determined from the von Mises yield criterion, as follows:

$$
\sigma_{t}^{\mathrm{Li}_{x} \mathrm{Si}}=\sigma_{\mathrm{n}}-\sigma_{\mathrm{Y}}
$$

From the force equilibrium and displacement constraint from the gap, the tangential stress at the interface in crystalline $\mathrm{Si}$ is then expressed as:

$$
\sigma_{t}^{\mathrm{Si}}=\frac{t_{\mathrm{Li}_{x} \mathrm{Si}}}{t_{\mathrm{Si}}} \sigma_{t}^{\mathrm{Li}_{x} \mathrm{Si}}=\frac{t_{1}+0.5 g}{t_{0}-t_{1}} \sigma_{t}^{\mathrm{Li}_{x} \mathrm{Si}}=\frac{t_{1} / t_{0}+0.5 g / t_{0}}{1-t_{1} / t_{0}} \sigma_{t}^{\mathrm{Li}_{x} \mathrm{Si}}
$$

Then, the mean stresses in crystalline $\mathrm{Si}$ and $\mathrm{Li}_{x} \mathrm{Si}$ at the interface would be given as:

After contact, $\{110\}$ :

$$
\sigma_{\mathrm{m}}^{\mathrm{Si}}=\frac{2 \sigma_{t}^{\mathrm{Si}}+\sigma_{\mathrm{n}}}{3}, \quad \sigma_{\mathrm{m}}^{\mathrm{Li}_{x} \mathrm{Si}}=\frac{2 \sigma_{t}^{\mathrm{Li}_{x} \mathrm{Si}}+\sigma_{\mathrm{n}}}{3}
$$

where, as shown below, $\sigma_{\mathrm{n}}$ is a negative quantity. Considering the limitation that the surface displacement due to swelling of $\mathrm{Li}_{x} \mathrm{Si}$ along the $<110>$ direction is equal to half of the initial gap, $g / 2$ and assuming that elastic deformation accommodates further growth of the layer and that lateral flow of $\mathrm{Li}_{x} \mathrm{Si}$ is suppressed, the normal stress $\left(\sigma_{\mathrm{n}}\right)$ that develops after contact as a function of the extent of continued lithiation may be estimated using a simple uniaxial stress analysis. For this analysis the dimension $t_{1}$, the thickness of the consumed Si layer, may be taken as a measure of the extent of lithiation. As shown in the Supplementary Note 1, the axial stress that develops after contact can be calculated as:

$$
\begin{gathered}
\sigma_{\mathrm{n}}=-\left[E_{\mathrm{Si}_{1}} E_{\mathrm{Li}_{x} \mathrm{Si}}\left(3 \frac{t_{1}}{t_{0}}-\frac{g}{2 t_{0}}\right)\right] /\left[E_{\mathrm{Li}_{x} \mathrm{Si}}+\left(4 E_{\mathrm{Si}}-E_{\mathrm{Li}_{x} \mathrm{Si}}\right) \frac{t_{1}}{t_{0}}\right], \\
\text { when } 3 \frac{t_{1}}{t_{0}} \geq \frac{g}{2 t_{0}}
\end{gathered}
$$


where $E_{\mathrm{Si}}$ and $E_{\mathrm{LixSi}}$ are Young's modulus of crystalline $\mathrm{Si}$ and $\mathrm{Li}_{x} \mathrm{Si}$, respectively.

In the estimation of the stress, the considered yield strength of $\mathrm{Li}_{x} \mathrm{Si}\left(\sigma_{Y}\right)$ is $1.0 \mathrm{GPa}$, and $E_{\mathrm{Si}}$ and $E_{\mathrm{Li}_{x} S \mathrm{Si}}$ are 180 and $35 \mathrm{GPa}$, respectively 15,16 . The ratio of the gap and initial thickness of crystalline $\mathrm{Si}\left(g / t_{0}\right)$ are $0.3,0.6,1.2$, and 2.4 . Figure $3 \mathrm{c}-\mathrm{e}$ show tangential, normal, and mean stresses and the change of free energy due to mechanical stress versus the extent of lithiation $\left(t_{1} / t_{0}\right)$ when $g / t_{0}$ is 0.3 (see also Supplementary Fig. 3a-d). The normal stress $\left(\sigma_{\mathrm{n}}\right)$ acting in both crystalline $\mathrm{Si}$ and $\mathrm{Li}_{x} \mathrm{Si}$ rapidly becomes more compressive after the contact (red solid line in Fig. 3c). The compressive tangential stress at the interface in $\mathrm{Li}_{x} \mathrm{Si}$ $\left(\sigma_{\mathrm{t}}^{\mathrm{L}} \mathrm{i}_{\mathrm{i}} \mathrm{Si}\right)$ develops after the contact together with the compressive normal stress (dashed line in Fig. 3c). The tangential stress at the interface in crystalline $\mathrm{Si}\left(\sigma_{\mathrm{t}}^{\mathrm{Si}}\right)$ rapidly increases after the contact as the tangential stress at the interface in $\mathrm{Li}_{x} \mathrm{Si}$ decreases (black solid line in Fig. 3c). The mean stresses at the interfaces in crystalline $\mathrm{Si}$ and $\mathrm{Li}_{x} \mathrm{Si}\left(\sigma_{\mathrm{m}}^{\mathrm{Si}}\right.$ and $\left.\sigma_{\mathrm{m}}^{\mathrm{Li}_{x} \mathrm{Si}}\right)$ for a given extent of lithiation $\left(t_{1} / t_{0}\right)$ are calculated by equations (3-8) as shown in Fig. $3 \mathrm{~d}$. $\sigma_{\mathrm{m}}^{\mathrm{Li}_{x} \mathrm{Si}}$ is constant before contact and $\sigma_{\mathrm{m}}^{\mathrm{Si}}$ increases slightly due to the increase of the tangential stress in crystalline $\mathrm{Si}$ on lithiation (see also Supplementary Fig. 3c). After contact, $\sigma_{\mathrm{m}}^{\mathrm{Li}_{\mathrm{i}} \mathrm{Si}}$ becomes more compressive following the trend of the normal stress. Assuming that $\Omega^{\mathrm{ii}} \times \Omega^{\mathrm{Si}} / \Omega^{\mathrm{Si}}$ is 4 and $x$ is $3.75\left(\mathrm{Li}_{3.75} \mathrm{Si}\right)$ considering a $400 \%$ volume change for fully lithiated $\mathrm{Si}$ at room temperature, Figure $3 e$ shows the change of free energy due to mechanical stress at the interface $\left(\Delta G_{\sigma}\right)$ for the extents of lithiation $\left(t_{1} / t_{0}\right)$ corresponding to the mean stresses shown and explains how mechanical clamping along the $\langle 110\rangle$ direction suppresses the lithiation of crystalline $\mathrm{Si}$ at the interface. Before the contact, $\Delta G_{\sigma}$ slightly increases from 0.09 to $0.094 \mathrm{eV}$ and the lithiation along $\langle 110\rangle$ direction is continued spontaneously since the free energy of $\mathrm{Li}$ deposition versus lithiation of $\mathrm{Si}$ $\left(\Delta G_{r}^{\mathrm{Ii}_{i}}-\mathrm{Li}_{x} \mathrm{Si}\right)$ is $0.18 \mathrm{eV}^{40}$. After the contact, as $\sigma_{\mathrm{n}}$ and $\sigma_{t}^{\mathrm{Li} x \mathrm{Si}}$ become more compressive, the increasing $\Delta G_{\sigma}$ reduces the gap of the net driving force between the lithiation of $\mathrm{Si}$ and $\mathrm{Li}$ deposition. Then, finally, $\Delta G_{\sigma}$ exceeds $\Delta G_{r}^{\mathrm{Li}-\mathrm{Li}_{x} \mathrm{Si}}$ as marked as a red dot in Fig. 3e and lithiation of $\mathrm{Si}^{\mathrm{r}}$ is stopped at the interface where the physical interaction induces a sufficiently big compressive normal stress $(<110>$ direction in the experiment). After this point is reached $\mathrm{Si}$ is lithiated mainly along the other direction, free from the physical contact $(<100>$ direction in the experiment). From the point of contact to the point at which the reaction is stopped the extent of lithiation $\left(t_{1} / t_{0}\right)$ changes by only 0.0025 (red dashed line), which means that mechanical contact can effectively prohibit further lithiation right after the contact is made. For larger gaps, the lithiation after contact goes further but is still less than $0.4 \%$ (see Supplementary Fig. 3d).

Mechanical fracture. Mechanical clamping of a Si structure on lithiation affects the fracture behaviour as well as the preferred direction of lithiation. Figure 4 shows how mechanical clamping enhances the fracture resistance of the lithiated Si pillar. The unclamped Si pillar has a critical diameter of $\sim 300 \mathrm{~nm}$ for fracture and the fracture ratio is almost $100 \%$ when its diameter is $>300 \mathrm{~nm}^{6}$. However, the clamped $<110>$ Si pillar with a diameter of $1 \mu \mathrm{m}$ and a gap of $300 \mathrm{~nm}$ expands along the unclamped $<100\rangle$ directions and only a few pillars show noticeable cracking after lithiation (Fig. 4a,b). But the clamped pillar shows size dependent fracture on lithiation just as the unclamped pillar does. When the diameter of the pillars increases to $2.2 \mu \mathrm{m}$ with a $300 \mathrm{~nm}$ gap, the pillars still show expansion along $<100\rangle$ direction but then significant cracks are found between $<110\rangle$ and $<100>$ directions (Fig. $4 \mathrm{c}, \mathrm{d}$ ). The statistical study of fracture ratio of the pillars can clearly show
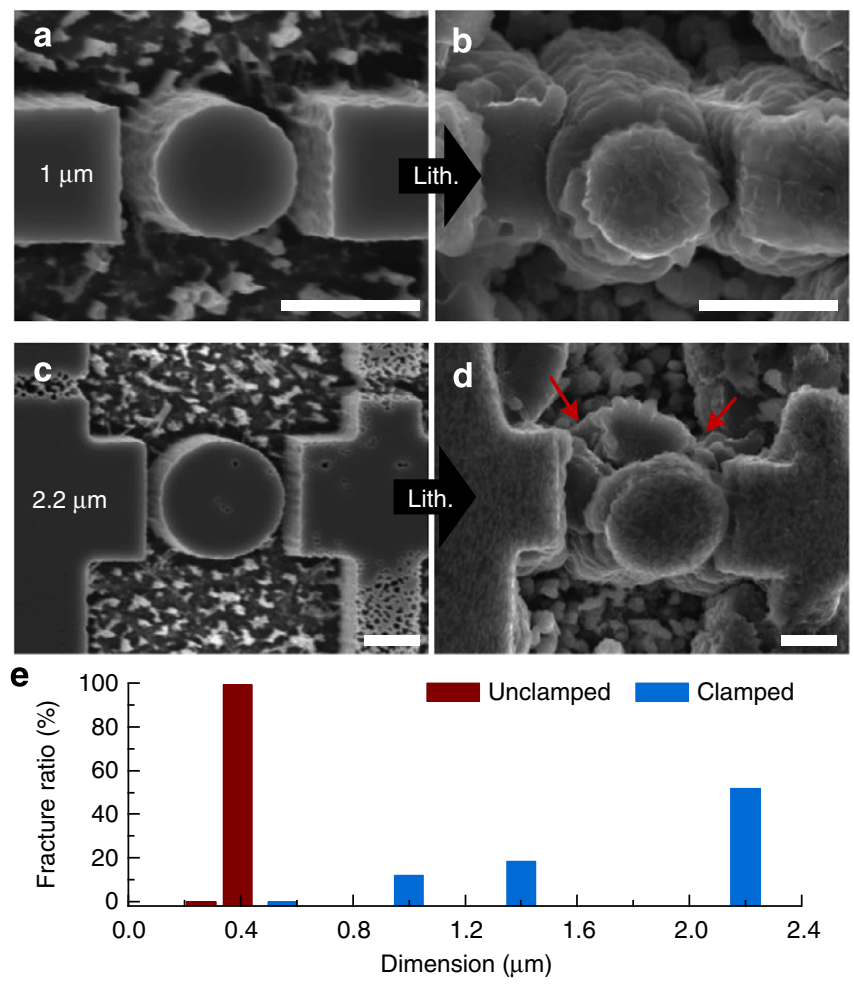

f

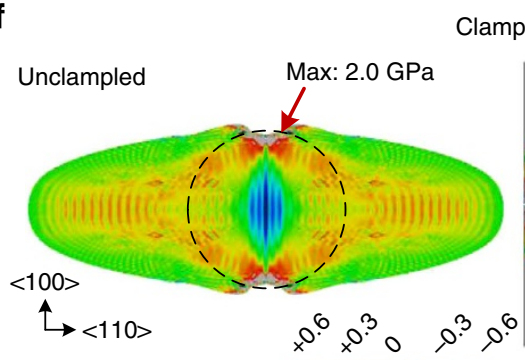

In-plane principle (GPa)

g

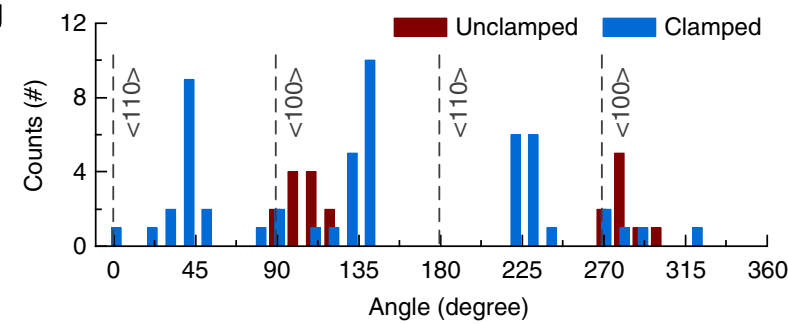

Figure 4 | Improved fracture resistance of the clamped Si nanopillar on lithiation. (a,b) SEM images of crystalline $<110>$ Si pillar of 1- $\mu$ m diameter and the walls with gap of $300 \mathrm{~nm}$. The pillar is clamped by the walls and expanded along $<100>$ direction upon lithiation. Significant crack is not found. (c,d) SEM images of crystalline $<110>$ Si pillar of $2.2 \mu \mathrm{m}$ diameter and the walls with gap of $300 \mathrm{~nm}$. After lithiation, the cracks are found between $<110>$ and $<100>$ directions as indicated by red arrows. Scale bars, $1 \mu \mathrm{m}$. (e) Column chart of the fracture ratio of the clamped $<110>\mathrm{Si}$ pillars for various diameters. To compare the effect of mechanical clamping for the fracture resistance, the fracture ratio of unclamped $<110>$ pillar is shown as red columns ${ }^{20}$. (f) Finite element analysis of in-plane principal stress of unclamped (left) and clamped (right) $<110>$ Si pillar after full lithiation. Initial diameter is $550 \mathrm{~nm}$ (dot circle) and lateral displacement of clamped pillar is confined to $160 \mathrm{~nm}$ (solid line). (g) Column chart of the population of the fracture location as an angle of the crack in the clamped $<110>$ Si pillar upon lithiation (blue). The population of the fracture location of the unclamped $<110>$ pillar (red) compares how mechanical clamping changes the fracture behaviour ${ }^{6}$. 
different fracture resistance for clamped and unclamped cases (Fig. 4e). The fracture ratio is obtained by counting the number of fractured pillars with various diameters (see Supplementary Fig. 4). The unclamped pillar shows a significant increase of fracture ratio from 0 to $99 \%$ when the diameter increases from 0.26 to $0.39 \mu \mathrm{m}$, as reported in our previous study ${ }^{20}$. In contrast, here the fracture ratio is $0 \%$ when the diameter of the clamped pillar is $0.55 \mu \mathrm{m}$ and only gradually increases as the diameter increases. When the diameters of clamped pillars are 1, 1.4, and $2.2 \mu \mathrm{m}$, the fracture ratios are $12 \%, 19 \%$, and $52 \%$, respectively. The diameter of the largest pillar in the test is about seven times of critical diameter of the unclamped pillar for fracture, but half of them have not fractured.

A finite element analysis can be used to explain how mechanical clamping affects the stress distribution and enhances fracture resistance of the Si pillar on lithiation. For this analysis the initial diameter of the simulated $<110\rangle$ Si pillar is $550 \mathrm{~nm}$ (dashed circle) and the gap between the pillar and the wall is $160 \mathrm{~nm}$ (see Fig. 4f). For the lithiation, the artificial moving boundaries between crystalline $\mathrm{Si}$ and $\mathrm{Li}_{x} \mathrm{Si}$ have a marching speed ratio of 5:1 along $\langle 110\rangle$ and $\langle 100\rangle$ directions, respectively, as in our previous analysis ${ }^{30}$ (see Supplementary Fig. 5). For the clamped pillar, the movement of the interface along the $<110\rangle$ direction is forced to stop after full contact is made (contact area does not increase). The volume change of lithiated $\mathrm{Si}$ is $400 \%$ and the considered mechanical properties are same with the analysis above (see Supplementary Note 2 and Supplementary Table 1). Figure $4 \mathrm{f}$ compares the estimated inplane principal stress of a fully lithiated Si pillar with/without mechanical clamping. As our previous studies have shown, the unclamped pillar shows a concentration of tensile stress as high as $2 \mathrm{GPa}$ on the top and bottom of the pillar along the $<100>$ direction ${ }^{20}$. The clamped pillar shows the concentration of tensile stress on the surface of the pillar along the diagonal direction between $<110\rangle$ and $<100>$ after the contact with the wall (see also Supplementary Movies 6 and 7). But the maximum tensile stress for the clamped pillar is only as high as $1.2 \mathrm{GPa}$. The lower maximum tensile stress for the clamped pillar compared with that for the unclamped pillar is caused by the compressive stresses associated with mechanical clamping, which leads to an enhanced fracture resistance, as shown in the experiment (Fig. 4e). The mechanical clamping also changes the fracture location. The statistical study of the population of crack locations on the pillar (Fig. 4f) shows that the favoured fracture site of the clamped pillar is located along a diagonal between the $<110>$ and $<100>$ directions (see Supplementary Fig. 6).

\section{Discussion}

Fundamental studies of $\mathrm{Si}$ as a negative electrode material for electrochemical reactions with $\mathrm{Li}$ have revealed how the mechanical stress caused by the large volume changes associated with the reaction plays an important role in both control of the reaction and fracture of the Si structures. However, while most studies have focused on the mechanical behaviour of individual Si particles, wires or pillars, $\mathrm{Si}$ anodes in batteries are composed of clusters of particles or wires of different shapes all in a confined space. In the present work, ex situ SEM and in situ TEM techniques were used to study the effects of mechanical interactions of well-defined crystalline Si nanopillar pairs during lithiation and how those interactions affect both the reaction kinetics and the fracture behaviour. When the Si structure is mechanically clamped by adjacent rigid walls along $<110>$ directions, the reaction in that direction is suppressed by compressive stresses that reduce the driving force for lithiation in that direction. This causes lithiation to occur in the transverse,
$<100>$, direction which is not favoured for unconstrained particles, wires or pillars. On the basis of our observations, we can imagine that the overall lithiation behaviour of real electrodes involve the swelling Si particles that push each other and translate to empty space until clamped conditions are reached. After the clamping of the most favoured lithiation directions, the reactions at the contact points are suppressed by compressive stresses and the other directions free from the clamping are consequently lithiated, much like filling the empty space (see Supplementary Fig. 7). Mechanical clamping of lithiated Si also markedly enhances the fracture resistance and increases the critical size for fracture because compressive stresses at the contact point compensate the concentrated tensile stress at the free surface. Thus, we can anticipate that the Si particles in the clusters in Li-ion batteries become more resistant to fracture than the individual $\mathrm{Si}$ structures that have received most attention. Although compressive stresses enhance the fracture resistance and promote filling of the empty space in the Si particle clusters, a space considering $400 \%$ volume change to allow complete lithiation is required to use maximum charge capacity of $\mathrm{Si}$ anode. Hence, further investigation is necessary to optimize the particle size and the empty space preventing mechanical fracture as well as allowing complete lithiation. In addition, since pristine crystalline Si remains amorphous after the first lithiation, the study of mechanical interaction of amorphous $\mathrm{Si}$ during electrochemical reaction is also demanded. Nevertheless, we believe that this study of mechanical interaction of lithiated $\mathrm{Si}$ pillars provides better idea of how Si structure will be studied and designed in the electrode level for high-performance Li-ion batteries.

\section{Methods}

Fabrication of Si nanopillar. $<110>$ crystalline Si pillar with walls was fabricated by e-beam lithography and dry etching (see Supplementary Fig. 1). Poly(methyl methacrylate) (PMMA) pattern for the mask of dry etching was defined on $<110>$ single crystalline Si wafer by e-beam lithography (Nova NanoSEM 450 Scanning Electron Microscope, FEI). Then, the $\mathrm{Si}$ wafer is etched by deep reactive ion etching (Deep RIE) process for $10 \sim 15$ min with $\mathrm{SF}_{6}$ gas for etching and $\mathrm{C}_{4} \mathrm{H}_{8}$ gas for passivation (Surface Technology Systems Co.). Finally, acetone and methanol cleaning removed PMMA pattern on the etched Si pillar and walls. For in situ TEM study, Si wafer was cut along $<110>$ direction by K\&S 775 Wafer Dicing Saw and PMMA pattern was defined on the cutting edge of the wafer (see Supplementary Fig. 2). The last fabrication process was same as mentioned above.

Electrochemical characterization by use of ex situ SEM. A piece of Si wafer with the pillar and wall structures as a working electrode was assembled with a polymer separator (Nagase \& Co. Ltd) and Li metal foil as a counter and reference electrode to build a sandwich structure of a half cell (see Supplementary Fig. 1i). BioLogic VMP3 multichannel battery tester-swept voltage of the cell down to $10 \mathrm{mV}$ versus $\mathrm{Li} / \mathrm{Li}^{+}$with a scan rate of $0.1 \mathrm{mV} \mathrm{s}^{-1}$ and it is was held for $>10 \mathrm{~h}$ for complete lithiation of the pillars. After the lithiation, the cell was disassembled and the electrode containing lithiated pillars was washed with acetonitrile to remove residual electrolyte in Ar-filled glove box. The sample was sealed in a vial in the glove box to avoid the oxidation of the sample and transferred to the vacuum chamber in SEM within $15 \mathrm{~s}$.

In situ TEM observation. The in situ electrochemical test was carried out in an FEI Titan 80-300 environmental TEM at the acceleration voltage of $300 \mathrm{kV}$. Nanofactory Instruments Dual-Probe STM-TEM in situ sample holder was employed to apply bias between Si nanopillars and Li metal counter electrode. During transferring the $\mathrm{Li}$ metal electrode inside TEM, the electrode was exposed to air for about $5 \mathrm{~s}$ to create a thin $\mathrm{Li}_{2} \mathrm{O}$ layer of about $20 \mathrm{~nm}$ functioning as a solid electrolyte. A relative bias of -4 or $-5 \mathrm{~V}$ was applied between the two electrodes, which caused $\mathrm{Li}^{+}$ions to be transferred to $\mathrm{Si}$ nanopillar electrode through the electrolyte.

\section{References}

1. Chan, C. K. et al. High-performance lithium battery anodes using silicon nanowires. Nat. Nanotech. 3, 31-35 (2008).

2. Wu, H. \& Cui, Y. Designing nanostructured Si anodes for high energy lithium ion batteries. Nano Today 7, 414-429 (2012). 
3. Whittingham, M. S. Materials challenges facing electrical energy storage. MRS Bull. 33, 411-421 (2008).

4. McDowell, M. T., Lee, S. W., Nix, W. D. \& Cui, Y. Understanding the lithiation of silicon and other alloying anodes for lithium-ion batteries. Adv. Mater. 25, 4966-4985 (2013).

5. Kasavajjula, U., Wang, C. \& Appleby, a. J. Nano- and bulk-silicon-based insertion anodes for lithium-ion secondary cells. J. Power Sources 163, 1003-1039 (2007).

6. Lee, S. W., McDowell, M. T., Berla, L. a., Nix, W. D. \& Cui, Y. Fracture of crystalline silicon nanopillars during electrochemical lithium insertion. Proc. Natl Acad. Sci. USA 109, 4080-4085 (2012).

7. Wu, H. et al. Stable cycling of double-walled silicon nanotube battery anodes through solid-electrolyte interphase control. Nat. Nanotech. 7, 310-315 (2012).

8. Liu, N. et al. A yolk-shell design for stabilized and scalable Li-ion battery alloy anodes. Nano Lett. 12, 3315-3321 (2012).

9. Liu, N. et al. A pomegranate-inspired nanoscale design for large-volumechange lithium battery anodes. Nat. Nanotech. 9, 187-192 (2014).

10. Magasinki, A. et al. High-performance lithium-ion anodes using a hierarchical bottom-up approach. Nat. Mater. 9, 353-358 (2010).

11. Yao, Y. et al. Interconnected silicon hollow nanospheres for lithium-ion battery anodes with long cycle life. Nano Lett. 11, 2949-2954 (2011).

12. Ge, M. et al. Large-scale fabrication, 3D tomography, and lithium-ion battery application of porous silicon. Nano Lett. 14, 261-268 (2014).

13. Shenoy, V. B., Johari, P. \& Qi, Y. Elastic softening of amorphous and crystalline Li-Si Phases with increasing Li concentration: A first-principles study. J. Power Sources 195, 6825-6830 (2010).

14. Zhao, K. et al. Lithium-assisted plastic deformation of silicon electrodes in lithium-ion batteries: a first-principles theoretical study. Nano Lett. 11, 2962-2967 (2011).

15. Sethuraman, V. a., Chon, M. J., Shimshak, M., Srinivasan, V. \& Guduru, P. R. In situ measurements of stress evolution in silicon thin films during electrochemical lithiation and delithiation. J. Power Sources 195, 5062-5066 (2010).

16. Ratchford, J. B. et al. Young's modulus of polycrystalline Li22Si5. J. Power Sources 196, 7747-7749 (2011).

17. Hertzberg, B., Benson, J. \& Yushin, G. Ex-situ depth-sensing indentation measurements of electrochemically produced Si-Li alloy films. Electrochem. Commun. 13, 818-821 (2011).

18. Pharr, M., Suo, Z. \& Vlassak, J. J. Measurements of the fracture energy of lithiated silicon electrodes of Li-ion batteries. Nano Lett. 13, 5570-5577 (2013).

19. Huggins, R. A. \& Nix, W. D. Decrepitation model for capacity loss during cycling of alloys in rechargeable electrochemical systems. Ionics 6, 57-63 (2000)

20. Ryu, I., Lee, S. W., Gao, H., Cui, Y. \& Nix, W. D. Microscopic model for fracture of crystalline Si nanopillars during lithiation. J. Power Sources 255, 274-282 (2014)

21. Zhao, K. et al. Concurrent Reaction and Plasticity during Initial Lithiation of Crystalline Silicon in Lithium-Ion Batteries. J. Electrochem. Soc. 159, A238-A243 (2012).

22. Yang, H. et al. A chemo-mechanical model of lithiation in silicon. J. Mech. Phys. Solids 70, 349-361 (2014).

23. Pharr, M., Zhao, K., Wang, X., Suo, Z. \& Vlassak, J. J. Kinetics of initial lithiation of crystalline silicon electrodes of lithium-ion batteries. Nano Lett. 12, 5039-5047 (2012).

24. Ryu, I., Choi, J. W., Cui, Y. \& Nix, W. D. Size-dependent fracture of Si nanowire battery anodes. J. Mech. Phys. Solids 59, 1717-1730 (2011).

25. Beaulieu, L. Y., Hatchard, T. D., Bonakdarpour, A., Fleischauer, M. D. \& Dahn, J. R. Reaction of Li with alloy thin films studied by in situ AFM. J. Electrochem. Soc. 150, A1457-A1464 (2003).

26. Rhodes, K., Dudney, N., Lara-Curzio, E. \& Daniel, C. Understanding the degradation of silicon electrodes for lithium-ion batteries using acoustic emission. J. Electrochem. Soc. 157, A1354-A1360 (2010)

27. Li, J. \& Dahn, J. R. An in situ X-ray diffraction study of the reaction of Li with crystalline Si. J. Electrochem. Soc. 154, A156-A161 (2007)

28. Chon, M. J., Sethuraman, V. A., McCormick, A., Srinivasan, V. \& Guduru, P. R. Real-time measurement of stress and damage evolution during initial lithiation of crystalline silicon. Phys. Rev. Lett. 107, 045503 (2011).
29. Goldman, J. L., Long, B. R., Gewirth, A. a. \& Nuzzo, R. G. Strain anisotropies and self-limiting capacities in single-crystalline 3D silicon microstructures: models for high energy density lithium-ion battery anodes. Adv. Funct. Mater 21, 2412-2422 (2011).

30. Lee, S. W., McDowell, M. T., Choi, J. W. \& Cui, Y. Anomalous shape changes of silicon nanopillars by electrochemical lithiation. Nano Lett. 11, 3034-3039 (2011).

31. Lee, S. W., Berla, L. a., McDowell, M. T., Nix, W. D. \& Cui, Y. Reaction front evolution during electrochemical lithiation of crystalline silicon nanopillars. Isr J. Chem. 52, 1118-1123 (2012).

32. Nam, S. H. et al. Probing the lithium ion storage properties of positively and negatively carved silicon. Nano Lett. 11, 3656-3662 (2011).

33. Berla, L. a., Lee, S. W., Ryu, I., Cui, Y. \& Nix, W. D. Robustness of amorphous silicon during the initial lithiation/delithiation cycle. J. Power Sources 258, 253-259 (2014).

34. Liu, X. H. \& Huang, J. Y. In situ TEM electrochemistry of anode materials in lithium ion batteries. Energy Environ. Sci. 4, 3844-3860 (2011).

35. McDowell, M. T. et al. Studying the kinetics of crystalline silicon nanoparticle lithiation with in situ transmission electron microscopy. Adv. Mater. 24, 6034-6041 (2012).

36. Liu, X. H. et al. In situ atomic-scale imaging of electrochemical lithiation in silicon. Nat. Nanotech. 7, 749-756 (2012).

37. Liu, X. H. et al. Ultrafast electrochemical lithiation of individual Si nanowire anodes. Nano Lett. 11, 2251-2258 (2011).

38. Liu, X. H. et al. Size-dependent fracture of silicon nanoparticles during lithiation. ACS Nano 6, 1522-1531 (2012).

39. McDowell, M. T. et al. In situ TEM of two-phase lithiation of amorphous silicon nanospheres. Nano Lett. 13, 758-764 (2013).

40. Limthongkul, P., Jang, Y.-I., Dudney, N. J. \& Chiang, Y.-M. Electrochemicallydriven solid-state amorphization in lithium-silicon alloys and implications for lithium storage. Acta Mater. 51, 1103-1113 (2003).

\section{Acknowledgements}

Y.C. acknowledges the support from the Assistant Secretary for Energy Efficiency and Renewable Energy, Office of Vehicle Technologies of the US Department of Energy. H.-W.L. acknowledges support from the Basic Science Research Program through the National Research Foundation of Korea (NRF) funded by the Ministry of Education, Science and Technology under NRF-2012R1A6A3A03038593. I.R. and H.G. acknowledge funding from the US Department of Energy through the DOE EPSCoR Implementation grant no. DE-SC0007074. W.D.N. gratefully acknowledges support from the Office of Science, Office of Basic Energy Sciences, of the U.S. Department of Energy under Contract No. DE-FG02-04ER46163.

\section{Author contributions}

S.W.L., H.-W.L., and Y.C. conceived the idea, designed experiments, analysed data and wrote the paper. S.W.L. carried out experiments. H.-W.L conducted in situ TEM characterization. I.R. did numerical analysis. W.D.N. and H.G. gave comments of mechanics. All the authors read the paper and made comments.

\section{Additional information}

Supplementary Information accompanies this paper at http://www.nature.com/ naturecommunications

Competing financial interests: The authors declare no competing financial interests

Reprints and permission information is available online at http://npg.nature.com/ reprintsandpermissions/

How to cite this article: Lee, S.W. et al. Kinetics and fracture resistance of lithiated silicon nanostructure pairs controlled by their mechanical interaction. Nat. Commun 6:7533 doi: 10.1038/ncomms8533 (2015).

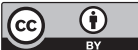

This work is licensed under a Creative Commons Attribution 4.0 International License. The images or other third party material in this article are included in the article's Creative Commons license, unless indicated otherwise in the credit line; if the material is not included under the Creative Commons license, users will need to obtain permission from the license holder to reproduce the material. To view a copy of this license, visit http://creativecommons.org/licenses/by/4.0/ 